\title{
Y a-t-il une exception paysanne ? : réalité et limites de la violence contestataire des paysans bretons
}

Nathalie Duclos

\section{(2) OpenEdition}

\section{Journals}

Édition électronique

URL : http://journals.openedition.org/conflits/209

DOI : $10.4000 /$ conflits.209

ISSN : $1777-5345$

Éditeur :

CCLS - Centre d'études sur les conflits lilberté et sécurité, L'Harmattan

Édition imprimée

Date de publication : 15 mai 1993

ISSN : 1157-996X

\section{Référence électronique}

Nathalie Duclos, «Y a-t-il une exception paysanne ? : réalité et limites de la violence contestataire des paysans bretons », Cultures \& Conflits [En ligne], 09-10 | printemps-été 1993, mis en ligne le 13 mars 2006, consulté le 30 mars 2021. URL : http://journals.openedition.org/conflits/209 ; DOI : https:// doi.org/10.4000/conflits.209

Ce document a été généré automatiquement le 30 mars 2021

Creative Commons License 


\title{
Y a-t-il une exception paysanne? : réalité et limites de la violence contestataire des paysans bretons
}

\author{
Nathalie Duclos
}

Trois exemples de reportages consacrés par la presse à des protestations paysannes

Manifestation du 2 octobre 1967 à Quimper. "En quelques minutes seulement, un commando fortement organisé, défonçait la porte d'entrée du local d'un député UNR. Les agriculteurs procédèrent à une véritable mise à sac, brisant les meubles, les jetant dans la rue...(Puis, ils) avancent vers la préfecture d'où sortent des CRS, qui chargent (..). On détruit des bancs, on abat les poteaux de signalisation, une DS brûle. Les manifestants mettent le feu au mobilier de la maison d'un gardien de la préfecture. Deux motos de la gendarmerie mobile sont incendiées (Arrivent les sapeurs-pompiers). Des manifestants assaillent la voiture, cassent les vitres, coupent les tuyaux, crèvent les pneus, malmènent les sapeurs-pompiers. La seconde voiture n'aura pas meilleur sort (..) Survient un camion de militaires. Le véhicule est cerné, les vitres cassées, les bâches déchirées. Les militaires sont arrachés de leurs sièges (..)"1. Au terme de ces affrontements, qui durèrent plusieurs heures, on dénombrera 179 blessés parmi les forces de l'ordre, dont 10 hospitalisés, l'un d'eux souffrant d'une fracture du crâne. Côté manifestants, on relèvera 80 blessés, dont 18 hospitalisés, l'un d'eux ayant également un traumatisme crânien. 17 juillet 1974 "Armés de barres de fer, des agriculteurs ont forcé les grilles d'un abattoir de Vannes et ont pénétré à l'intérieur des bâtiments, où ils ont découpé, scié et enlevé des carcasses d'animaux et arrosé de fuel les autres, soient 120 tonnes de viande. Puis des feux ont été allumés devant les grilles de la préfecture pour y brûler les carcasses. Des pierres, boulons et autres projectiles ont brisé fenêtres et vitres de la préfecture. Des manifestants ont également cassé, à 
l'aide de barres de fer, les parcmètres de la place. Après dispersion certains agriculteurs se sont rendus dans l'exploitation d'un éleveur-expéditeur, ont saccagé son étable, après que les bêtes aient été détachées. D'autres ont déposé un mouton mort dans la propriété de M. Grimaud, député-maire"2 22 avril 1983. "Les manifestants attaquent la prison de Quimper (..) Poursuivis par les CRS, ils déferlent dans la ville. Armés de barres de fer, ils arrachent des panneaux de signalisation, des corbeilles à papier, des feux tricolores. Puis c'est l'accalmie. (..) Revenant de la manifestation, une centaine d'agriculteurs ont envahi la sous-préfecture de Chateaulin. Deux gendarmes qui tentaient de raisonner des manifestants furent violemment pris à partie et ont dû être hospitalisés. Leur voiture a été jetée dans un canal. Les agriculteurs ont pénétré dans la sous-préfecture, après avoir brisé le rideau métallique du garage et la porte d'entrée à l'aide de barres à mine et de masses. Ils ont mis à sac plusieurs bureaux et incendié des dossiers"3. Ces reportages suffisent à montrer que la violence, définie au sens étroit comme "comportement visant à causer des blessures aux personnes ou des dommages aux biens" ${ }^{4}$, s'est manifestée avec éclat à l'occasion des contestations paysannes de ces trois dernières décennies. Alors que l'on sait, notamment depuis la manifestation de 1982 à Paris ${ }^{5}$, que les organisations syndicales d'agriculteurs sont en mesure d'organiser d'immenses démonstrations pacifiques, la décennie 80 n'a pas pour autant vu disparaître des protestations qui l'étaient moins. Peut-on considérer les mobilisations des paysans comme des "flambées" de mécontentement, donnant spontanément libre cours à tous les excès? La plupart des journalistes et chercheurs s'accordent sur le caractère spécifique, et persistant, des violences qui ponctuent leurs actions collectives. Il est vrai que l'on observe en France depuis trente ans, plus particulièrement dans le Midi viticole et en Bretagne ${ }^{6}$, des modalités de protestations particulièrement virulentes de la part de cette catégorie sociale. Les paysans seraientils irréversiblement violents dans leur contestation, et cela depuis les jacqueries de l'Ancien Régime ? On ne fera pas nôtre sans réexamen cette thèse d'une violence intemporelle. On objectera que rien ne pourrait à priori expliquer le fait que les agriculteurs ne subissent pas, comme l'ensemble de la population, le processus de "civilisation des moeurs"7 qui, dans le cadre des actions protestataires, se manifeste en longue période par une tendance à la modération de la violence. Des transformations sensibles ont affecté la catégorie sociale "agriculteurs" ces dernières années : scolarisation plus longue et meilleure formation, spécialisation des productions et plus grande intégration à l'économie. Il serait surprenant que cette évolution n'entraîne pas une modification des mobilisations paysannes, les modalités des protestations étant liées aux caractéristiques sociales de ceux qui les mettent en oeuvre. En réalité, trois reportages isolés, comme ceux évoqués précédemment, ne peuvent rien nous apprendre sur la fréquence du recours à la violence parmi les modes d'action paysans, ni sur leur éventuelle variation dans le temps. Aussi une analyse diachronique approfondie du répertoire ${ }^{8}$ paysan s'impose-t-elle, et cela aux fins de cerner d'éventuelles transformations de sa structure. Quelle place y occupent les actions violentes ? Y a-t-il réellement une exception paysanne dans le processus général de modération des luttes qui affectent les conflits sociaux ? Dans ce but, on a choisi d'étudier les mouvements ${ }^{9}$ paysans les plus violents qui se sont produits en Bretagne sous la $\mathrm{V}^{\circ}$ République, à partir de l'ensemble des comptes-rendus les concernant, parus dans Le Monde et dans Ouest-France. Ce corpus servira de support à la définition $\mathrm{d}^{\prime}$ indicateurs de violence ${ }^{10}$ et à l'établissement de typologies d'actions, qui permettront une analyse comparative. C'est à cette objectivation que nous consacrerons notre 
première partie, afin de prendre la mesure de la violence des paysans bretons. Puis nous dépasserons cette approche, pour nous intéresser à la dynamique des protestations paysannes et à leur système symbolique, dans le but de comprendre comment se modèlent les registres d'action en fonction des situations d'interdépendance stratégique.

\section{PERSISTANCE DES MODALITES VIOLENTES DE PROTESTATION}

3 Les actions collectives des paysans revêtent les formes les plus variées, et ne se laissent pas analyser d'emblée sans une classification minimale. Afin d'apprécier la configuration de ce répertoire et son éventuelle variation dans le temps, nous distinguons huit types d'actions. Au sein de la première catégorie manifestations nous classons tout ce qui est mentionné par les journalistes comme manifestation/défilé, rassemblement (regroupement concerté de paysans, sans défilé), ou meeting. La deuxième catégorie communication comprend toutes les actions qui visent à entrer en contact avec des populations extérieures au monde paysan (élus, pouvoirs publics, "opinion publique", etc.), afin d'évoquer ses préoccupations. Sous la troisième catégorie barrages se trouvent inclus non seulement les obstacles matériels à la circulation routière ou ferroviaire, mais aussi les piquets qui barraient l'accès à des sites (comme les industries laitières en 1972). On a précisé la finalité de ces barrages (arraisonnement ou non de véhicules), le nombre d'interceptions et le nombre de fois où le contenu des camions a été détruit. Egalement, on a indiqué si ces barrages occasionnaient des déprédations (rails arrachés, systèmes de signalisation SNCF détériorés, etc.), s'ils étaient filtrants ou, au contraire, provoquaient le blocus total d'une localité. Une quatrième catégorie inclut toutes les pénétrations (ou tentatives, contrariées par les forces de l'ordre) dans des lieux publics ou privés, en précisant si elles donnent lieu également à des occupations, déprédations ou séquestrations. Dans une cinquième catégorie, on a recensé les dégradations, déprédations et destructions, en les considérant respectivement comme: salissures ou souillures qui demanderont nettoyage; détériorations qui nécessiteront réparation, remise en état; actes provoquant l'anéantissement. La sixième catégorie concerne toutes les formes d'agressions ad hominem, c'est à dire des brutalités commises à l'encontre de personnes étrangères aux protestations paysannes (journalistes, automobilistes, etc.). La septième catégorie comprend les mises à sac, c'est à dire les opérations de destruction systématique d'un local. La dernière catégorie, d'une toute autre nature, puisqu'elle n'est pas directement le fait des paysans eux-mêmes, regroupe les interventions des forces de l'ordre, le nombre d'affrontements auxquelles elles ont donné lieu, et le nombre éventuel de blessés qu'il y a eus à ces occasions.

On peut dès lors dresser un "tableau récapitulatif du répertoire paysan de 1961 à 1990" : 4 ?419611964196719691972197419801983198419861990

MANIFESTATIONS 1243103210519837

COMMUNI-CATION 1442271217

BARRA-

GES 55523 inchiffra-ble 10585441922 


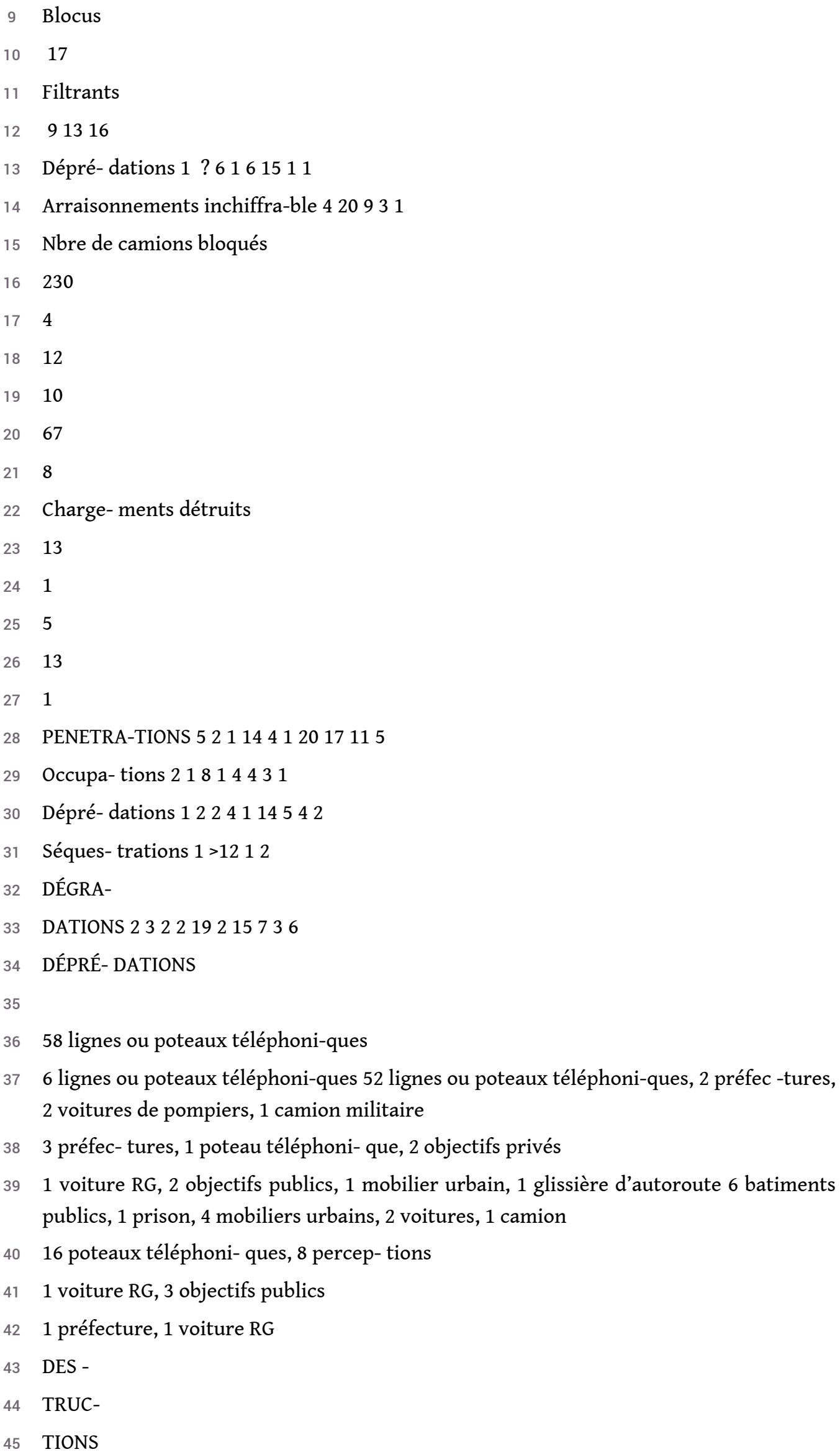


611

1

2

2

1

bulletins électo-raux : 3

carcas- ses de veaux : 2 mobilier urbain,

$1 \mathrm{DS}$,

2 motos de gendar-merie,

1 porte de préfecture, 1 prison

parcmè-tres

3 véhicules de police

2 véhicules

1 wagon, 1 voiture de police

1 banc public , 1 guérite de préfecture

AGRES- SIONS AD HO- MINEM

MISES À SAC 21423

INTERVENTIONS FORCES DE L'ORDRE 1052451311953

Affronte-ments 42323123

NOMBRE DE BLESSÉS

FO $: 5$

paysans : >1 FO : 192 (11 hospitali-sés) paysans : 92 (18 hospitali- sés)

paysans : > 2 (1 hos-pitalisé)

FO : 2 paysans : 5

2 gendar-mes hos-pitalisés paysans : 4

FO $: 1$ paysans : $>4$

1 CRS (par accident)

* Lorsque nous ne disposions pas de données chiffrées sur les actes indiqués au pluriel, nous avons retenu le chiffre 2 .

Quelle est l'importance de la violence au sein du répertoire paysan depuis 30 ans ? Pour répondre à cette question, il faut établir trois sous-catégories, parmi les types d'actions collectives que nous avons distingués précédemment, en fonction de la violence qui les caractérise. Au sein de la première, on rapproche les "manifestations" des opérations de "communication". Ces deux types d'actions sont en effet non-violentes, les premières étant une "expression pacifique d'une opinion ou d'une revendication"11, et les secondes tentant de persuader des tierces personnes du bien-fondé de la cause des organisateurs. Toute violence qui surviendrait à l'occasion de ces actions risquerait même de produire le contraire de l'effet recherché. Aussi sont-elles entourées de multiples précautions pour l'éviter ${ }^{12}$. La seconde sous-catégorie "actions de contrainte" regroupe les "pénétrations" et les "barrages" : bien que ces actions soient fondées sur 
l'usage de la contrainte physique, on les différencie des actions violentes car leur finalité n'est pas de provoquer des détériorations, destructions ou blessures ${ }^{13}$. Si, là encore, des violences peuvent se produire à l'occasion de leur mise en oeuvre, celles-ci risquent aussi assez sûrement de desservir les manifestants. On les distingue néanmoins des actions non violentes dans la mesure où elles se fondent sur la contrainte physique, et usent du registre symbolique dans une moins large mesure que les manifestations ou opérations de communication. La troisième sous-catégorie est constituée par les "dégradations", "déprédations" et "destructions", "agressions ad hominem" et "mises à sac". Seule cette catégorie d'actions collectives peut véritablement être considérée comme violente, dans la mesure où elle produit des détériorations, temporaires ou définitives, ou des blessures voire des morts. On peut discerner différentes modalités de violence, à partir de la distinction: violence instrumentale/violence colérique. On considérera que les actions collectives relèvent de la première notion, lorsqu'elles sont "exercée(s) en principe sans passion, en vue d'atteindre des objectifs assignés, et ce parce que ses auteurs savent par expérience qu'elle(s) ser(ont) efficace(s) dans cette perspective" ${ }^{14}$. Le recours à la violence fait alors l'objet de calculs: les actions de ce type sont choisies parmi un multiple de possibles. Elles sont insérées dans une stratégie de contestation et mises au service de la cause défendue. La violence colérique quant à elle est "une explosion de ressentiment actif ${ }^{\prime 15}$, qui suspend au moins partiellement le calcul coûts/avantages. Elle apparaît comme "le sous-produit d'actions et d'interactions qui manifestent une volonté d'agir en fonction d'intérêts contradictoires"16. Aussi survient-elle de manière plus aléatoire, selon des logiques produites par les situations dans lesquelles se déroulent les actions collectives. Cette distinction entre violence instrumentale et colérique nous permet de différencier les actions collectives en fonction de leur intentionnalité violente. On considérera comme indicateur de modération des protestations paysannes la diminution des actions intentionnellement violentes. Parmi les actions entreprises par les paysans bretons, les mises à sac relèvent du type instrumental. S'agissant des "agressions ad hominem", "destructions", "déprédations" et "dégradations", qui se produisent à l'occasion de manifestations, de barrages ou d'investissements, les informations dont nous disposons ne permettent pas toujours d'établir avec certitude leur appartenance à tel ou tel registre de violence. On remarquera néanmoins que les agressions ad hominem relèvent très probablement $\mathrm{du}$ type colérique. Pour la plupart des destructions, déprédations et dégradations commises à l'occasion de manifestations, il est difficile de statuer. Cependant, au sein de ces trois catégories, on peut sans hésitation considérer comme violences instrumentales les déprédations de lignes téléphoniques, les plasticages et les destructions opérées en 1961, 1964 et 1990. Un dernier type d'indicateur de violence a été laissé de côté jusqu'ici: les "contacts" entre paysans et forces de l'ordre. Cette catégorie est en effet difficilement prédictive de la violence paysanne. Les interventions des forces de l'ordre ne constituent pas nécessairement l'indice d'excès des manifestants, dans la mesure où elles risquent, plus sûrement, de témoigner du degré d'acceptation des désordres par les pouvoirs publics. Par contre, les affrontements entre paysans et forces de l'ordre peuvent être considérés comme un indicateur de la pugnacité des paysans et de leur propension à l'acceptation, voire à la recherche de contacts physiques. Aussi porterons-nous notre attention sur leur durée, ainsi que sur le nombre de blessés et la gravité de ces blessures, notamment du côté forces de l'ordre. Cette donnée est un indice particulièrement pertinent de la violence paysanne. A partir 
de cette distinction entre actions non violentes, contraignantes et violentes, quels enseignements peut-on tirer d'une perspective diachronique? Nous conclurons à une modération de la violence protestataire des paysans si, et seulement si, une évolution se produit, de façon conjuguée sur trois niveaux: moindre recours à des actions d'intentionnalité violente. Baisse de la fréquence des violences. Plus grande "acceptabilité" sociale des méthodes protestataires, parce qu'elles seraient orientées de moins en moins fréquemment vers les individus. Les mises à sac sont les seules catégories d'actions que l'on peut considérer avec certitude comme intentionnellement violentes. Or, elles sont présentes à chaque décennie, et n'ont pas connu d'évolution statistique notable. Leur récurrence jusqu'en 1984 nous amène à conclure à la persistance de modalités protestataires particulièrement violentes chez les paysans. Considérons maintenant les "déprédations", "destructions" et "dégradations". Si les "destructions" n'ont pas varié de manière significative après 1961, les "déprédations" quant à elles ont sensiblement décru depuis cette période: alors que des poteaux téléphoniques étaient sciés en grand nombre en 1961 et 1967, ces détériorations ont quasiment disparu depuis, (excepté en 1984, mais à une échelle moindre); les paysans s'en sont pris davantage aux façades de bâtiments publics ou privés, ou encore aux aménagements urbains, mais dans une bien moindre mesure qu'auparavant lorsqu'ils sciaient des lignes téléphoniques. Ces observations nous amènent à conclure que la fréquence de ce type de violences est désormais moindre. Pourtant, d'autres considérations viennent contredire cette première analyse. Les "dégradations" se sont en effet amplifiées en 1974 : elles étaient alors dix fois plus nombreuses que dans les années 60 , et n'ont sensiblement décru que depuis 1984. D'autre part, dans les années 70 , des modalités violentes de mise en oeuvre d'actions seulement contraignantes, sont apparues et ont persisté depuis, voire se sont accentuées. Ainsi, les "barrages et "pénétrations" offraient très rarement l'occasion aux paysans de commettre des déprédations, destructions ou séquestrations dans les années 60. Depuis 1972, toutes les "pénétrations" ont été accompagnées de déprédations, avec une forte pointe en 1983. A partir de cette même date, les "barrages" ont donné régulièrement lieu à des déprédations, avec cette fois une forte présence de ce type d'actions en 1984. Egalement, il est devenu fréquent que soient commises des destructions du contenu des véhicules arraisonnés, notamment en 1972, 1983 et 1984. On pourrait déduire de ces observations que les paysans sont désormais plus violents. Si l'on veut bien admettre que cette croissance des déprédations et destructions à l'occasion de "barrages" ou d'"investissements" équilibre, grosso modo, la baisse des détériorations de lignes téléphoniques ${ }^{17}$, on en arrive à conclure simplement à une persistance de la violence protestataire des paysans. Observe-t-on enfin une baisse des violences à l'encontre des individus? La réponse est négative en ce qui concerne les agressions ad hominem. Par contre, le nombre de blessés à l'occasion d'affrontements avec les forces de l'ordre nous fait pencher vers l'idée d'une modération de la violence dans les protestations paysannes. L'évolution est ici particulièrement sensible. Alors qu'on dénombrait 197 agents du maintien de l'ordre blessés dans les années 60, parmi lesquels 11 durent être hospitalisés, l'un d'eux souffrant d'un traumatisme crânien, "seuls" 6 CRS et gendarmes furent blessés dans les années 80, deux d'entre eux ayant été hospitalisés. Les statistiques concernant les paysans connaissent une évolution similaire: plus de 94 manifestants furent blessés dans les années 60, 18 étant hospitalisés, l'un d'eux ayant une fracture du crâne. Dans les années 80 , on n'en dénombrait plus "que" 13, dont un grièvement et au moins deux légèrement. Cette évolution statistique nous fait penser 
que les paysans évitent dorénavant davantage les affrontements physiques avec les forces de l'ordre. Ajoutons enfin que le répertoire paysan des mouvements de 1986 et 1990 témoigne véritablement d'une modération de leurs modalités d'action protestataire. On n'y observe notamment aucune violence de type instrumental, pas plus que des violences contre les individus. Pour autant, ces deux mouvements ne permettent pas, à eux seuls, de conclure à l'abandon de toute violence. Peu d'années auparavant, en 1983 et 1984, les paysans se sont exprimés de manière particulièrement virulente. Compte tenu de la stabilité générale des processus sociaux, et notamment des répertoires d'action, on ne saurait conclure que les mouvements de 1986 et 1990 sont significatifs d'une évolution vers la modération. Seule la diminution très sensible du nombre de blessés du côté forces de l'ordre laisse véritablement augurer une modération dans l'expression des protestations paysannes. Pour le reste, la récurrence des actions intentionnellement violentes et la fréquence toujours très grande des autres types violents de protestation conduisent à conclure à la "continuité du répertoire"18 paysan sous cet aspect. Pourtant, à partir d'un déplacement de perspective, qui sera centré cette fois sur les processus de contestation, on est amené à reconsidérer ces premières analyses.

\section{MODERATION TENDANCIELLE DES PROCESSUS DE CONTESTATION}

A partir de ces premiers résultats, l'hypothèse initiale selon laquelle il y aurait eu modération de la contestation paysanne semble loin d'être vérifiée. Elle paraitrait même totalement invalidée, voire contredite, notamment par la persistance d'actions comme les mises à sac, qui témoignent d'une instrumentalisation de la violence de la part des paysans. Pourtant, cette impression est fondée sur une approche qui introduit un biais dans l'analyse. En effet, ces conclusions sont tirées de l'observation d'actes envisagés séparément, dégagés, pour les commodités de l'analyse, du faisceau d'actions collectives que constitue chaque mouvement revendicatif. Or, ainsi que le fait remarquer Charles Tilly, au sein des "mouvements sociaux" que nous connaissons aujourd'hui, les "leaders tentent de lier organiquement et symboliquement des actions concrètes qui combinent différents éléments du nouveau répertoire" ${ }^{19}$. Aussi faut-il analyser les modes d'action paysans en les situant dans un processus global, et en donnant toute leur importance aux représentations afférentes aux actions entreprises. Dans cette perspective, nous ferons une analyse comparée de protestations paysannes anciennes et récentes, qui ont été les plus violentes de chaque période. L'étude portera d'abord sur la contestation de juin $1961^{20}$, dont la revendication essentielle concernait l'application de la loi d'orientation agricole, votée par le Parlement en $1960^{21}$. On retiendra également les conflits de 1983 et 1984, dont l'objectif était le démantèlement des montants compensatoires monétaires négatifs. L'approche quantitative des actions collectives engagées à l'occasion de ces mouvements ne laisse pas apparaître de différence significative entre leurs modalités respectives de protestation. Pourtant, la contestation de 1961, du fait de sa dynamique et du système de référence dans lequel elle a été conçue, se fondait presque uniquement sur l'usage de la force directe et l'instrumentalisation de la violence. En 1983 et 1984 par contre, les actions violentes ne constituent plus autant le registre dominant de la mobilisation. Elles sont désormais associées à des opérations de communication et de séduction, et laissent une plus 
grande place aux négociations, ce qui confère à ces contestations une tonalité moins virulente. Ainsi elles ne s'inscrivent pas dans une logique conflictuelle aussi dure qu'en 1961. La violence du mouvement de 1961 est beaucoup plus fondamentale que ne le laisse supposer l'observation isolée des actions collectives qui l'ont caractérisé. Tout d'abord, si l'on établit le rapport existant entre le nombre de jours au cours desquels des actions protestataires ont été mises en oeuvre, et la durée du conflit, on prend toute la mesure de l'intensité du mouvement de 1961. Sur 27 journées de conflit, 20 donnèrent lieu à des actions collectives, soit presque trois jours sur quatre. On affinera ce constat en distinguant deux périodes dans ce mouvement : la première constitue les prémisses d'un conflit dont l'investissement de la sous-préfecture de Morlaix le 8 juin, minutieusement préparé et parfaitement mis en scène ${ }^{22}$, marquera l'ouverture sur la scène publique. Si, avant le 8 juin, les actions ne sont que sporadiques, elles se multiplieront singulièrement ensuite, donnant l'impression d'un crescendo sans fin. Il ne se passe alors pas un jour sans que des poteaux téléphoniques ne soient sciés ou que des tracteurs n'obstruent les routes. Les paysans donnent ainsi à voir la montée en puissance d'une révolte d'autant plus profonde que les actions de protestation s'étendent du Finistère vers les autres départements bretons, et confèrent ainsi au mouvement une forme tournante. Dans cette mesure, la date du 22 juin, jour du jugement des deux principaux instigateurs de ce qui a été depuis constitué comme la "prise" de la sous-préfecture, devient un moment charnière, à partir duquel le mouvement semble devoir franchir un seuil nouveau d'intensité ou, au contraire, marquer le pas selon le résultat du jugement. Cette analyse est confirmée par Pierre Abéguilé, qui estime que l'acquittement de Gourvennec et Léon a permis de désamorcer un conflit devenu incontrôlable : "Il n'aurait pas fallu qu'ils restent en prison, il aurait pas fallu (..) s'ils avaient condamné, ç'aurait été terrible"23. La forme que prend la contestation est également significative d'une logique conflictuelle dure. Les paysans s'emploient en effet à harceler les pouvoirs publics, leur démarche étant sous-tendue par une représentation quasi guerrière du combat mené. Cela est perceptible à travers les détériorations de lignes téléphoniques, qui constituent l'essentiel de leur répertoire. A ces occasions, les paysans mettent en toute lucidité la violence au service de leur cause. Cette instrumentalisation de la violence participe d'une analogie avec la rébellion algérienne. En 1961, nombre de paysans ont fait, comme appelés du contingent, la guerre d'Algérie. Tirant les leçons du désarroi provoqué par les méthodes des fellaghas auprès des autorités françaises, ils vont recourir aux mêmes moyens. Le principal organisateur des protestations paysannes, Jean-Marie Saillour, parachutiste en Algérie, justifie cette importation : "Jusqu'au procès, c'était la guérilla ! On a scié les poteaux téléphoniques. C'était un peu ce que faisaient les fellaghas en Algérie aussi. En Algérie, toutes les nuits, les poteaux étaient coupés... Il fallait faire quelque chose. Ce n'était pas très populaire bien sûr, mais c'était un moyen de semer le désordre (..) Cela marquait, parce que cela créait une ambiance de désordre, de tension"24. Ces propos sont corroborés par ceux de Pierre Abéguilé, qui, en 1961, avait conscience du caractère performant de la guérilla menée par les nationalistes algériens: "A Morlaix, c'est moi qu'ai été désigné donc, pour dire "Faites tout ce que vous voulez. Coupez les poteaux téléphoniques". J'avais voulu faire référence à l'Algérie (..) en disant "là-bas, ça réussit", parce qu'on sentait que ça réussissait déjà (..) Pourquoi pas ici ?" ${ }^{25}$. Les modalités de mise en place des barrages conduisent également à réévaluer à la hausse leur potentiel de violence. Nombre d'entre eux, réalisés à l'aide de tracteurs, ne se limitaient pas à l'obstruction d'une route; postés sur toutes les voies 
d'accès à une localité, ils provoquaient son blocus pendant plusieurs heures. Les paysans, en opérant de la sorte, signifiaient de manière à peine voilée leur capacité à menacer l'ordre établi, à faire front face à la ville, localisation traditionnelle de l'Etat. Il $\mathrm{y}$ a dans ces modalités un emprunt indubitable aux techniques guerrières du siège, et affichage de la plausibilité d'un déploiement très élevé de violences, l'assaut étant suggéré comme dernière étape et finalité ultime du blocus. Ce caractère menaçant des protestations était renforcé par le fait qu'elles se produisaient très fréquemment de nuit. Cela était presque systématiquement le cas des détériorations de lignes téléphoniques, mais valait également pour les blocus de localités, mis généralement en place à l'aurore. De plus, ces actions étaient organisées dans le plus grand secret. Le caractère dynamique des mobilisations tient au fait que les paysans s'appuient à cette époque sur une organisation déjà bien rodée, qui a fait ses preuves, et à partir de laquelle ils savent pouvoir réaliser des manifestations massives et surprenantes. Depuis environ deux années, sous la houlette de jeunes paysans, les structures syndicales de la FDSEA du Finistère ont été profondément remodelées afin d'établir des liaisons beaucoup plus étroites entre la base et l'état-major. Cette rénovation est passée par un découpage nouveau, reposant sur les quartiers, communes et cantons, restructurant ainsi le syndicat de manière pyramidale. Le syndicalisme repose désormais sur la participation de la base aux prises de décision: chaque sujet de préoccupation est discuté en réunions, organisées à la plus petite échelle qui soit : le quartier. Quant aux décisions sur les mobilisations envisagées, elles sont prises à la suite d'un vote. Le quadrillage syndical ${ }^{26}$ témoigne d'une logique quasi guerrière. Les dirigeants du mouvement comptent sur l'effet produit par une levée en masse des paysans. Pour cela, il leur faut une discipline et une loyauté totale de leurs troupes. Le nouveau système de prise de décision est mis en place à cette fin. De fait, il apparaît comme une véritable machine de guerre au service de l'état-major syndical. A propos de l'investissement de la sous préfecture de Morlaix, l'un d'eux le reconnaît : "Il y a eu un vote, mais on savait que le vote était acquis. Parce qu'il faut toujours donner l'impression que ce sont les autres qui décident" ${ }^{27}$. Forts de cette capacité à faire avaliser leurs décisions, les dirigeants syndicaux s'assurent de la discipline de leur base en faisant signer un engagement par chaque paysan, devant les autres agriculteurs de son quartier, afin que s'exerce un contrôle mutuel. Ils ont de ce fait une excellente maitrise de leur base. Par la suite, le "dispositif d'alerte" se met en branle : chaque paysan est chargé précisément de prévenir certains autres agriculteurs, lesquels vont à leur tour diffuser l'information. De la sorte, le syndicat était en mesure, dès janvier 1960, au début de sa mise en place, de mobiliser 15000 hommes en deux heures. Ainsi, en 1961, les actions collectives rassemblaient un nombre très élevé de paysans : chaque barrage réunissait sans difficulté 1000 à 3000 paysans avec plusieurs centaines de tracteurs. Ce n'est pas la révolte de quelques uns, mais celle unanime de la population paysanne que ses organisateurs voulaient donner à voir, et à croire. Par là, les paysans menaçaient les autorités d'un soulèvement général, qui aurait pu prendre une configuration des plus violentes. Enfin, les paysans se jouent de la capacité prédictive des mobilisations par les autorités: conscients de faire éventuellement l'objet de mise sur écoute par les Renseignements Généraux, ils ont proscrit totalement l'usage du téléphone de leur "dispositif d'alerte" ; ou, au contraire, s'ils l'utilisèrent, c'était afin de faire parvenir de fausses informations à la police et de perturber son organisation du maintien de l'ordre. Les "manifestations" semblaient ainsi pouvoir survenir à n'importe quel moment et n'importe où, selon le bon vouloir des organisateurs, sans que personne 
puisse avoir une quelconque prise sur eux. Les références identitaires des paysans attestent également du fondement violent de cette contestation. Des observateurs tels Pierre Viansson-Ponté, du Monde, ont fait remarquer que les succès des discours de meetings étaient dûs souvent "à une hostilité violente contre tout ce qui n'est pas paysan (commerçants, gens des villes, gouvernement, Etat) et évoquaient le dorgérisme d'avant-hier, et le poujadisme d'hier"28. De fait, les références au mouvement de Dorgères $^{29}$ sont présentes notamment avec les fourches, symbole du mouvement des chemises vertes, dessinées sur le bitume en plusieurs endroits. De la sorte, les paysans réactivaient la mythologie des jacqueries et tentaient d'inspirer au pouvoir de sérieuses craintes quant au caractère subversif de leur contestation. On retrouve également l'arrière-fond idéologique du poujadisme au travers de la revendication d'un "plan de Constantine" pour la Bretagne, adaptation régionale de la célèbre formule de TixierVignancour: "La Corrèze avant le Zambèze". Cette revendication d'un "plan de Constantine" est par ailleurs significative de l'analogie qui s'établit dans les représentations des paysans entre leur mouvement de contestation et la révolte algérienne de l'époque. A l'instar des nationalistes algériens, les Bretons s'estiment en rébellion contre le pouvoir central; d'où la dénomination de fellaghas qu'attribuaient à leurs troupes certains tribuns parmi les plus influents, comme Alexis Gourvennec. En aucune manière les protestations de 1983 et 1984 n'ont atteint une intensité aussi grande et n'ont donné les signes d'une semblable propension à la violence. Si les paysans ont également eu recours à l'action directe afin d'obtenir satisfaction de leurs revendications, cette démarche ne fut pas aussi systématique, les manifestants assortissant leurs actions les plus virulentes d'opérations jouant hautement sur le registre symbolique. Les protestations de cette période traduisent en effet une réelle tentative de verbalisation des motifs de mécontentement. Cette évolution tient aux transformations des contextes sociaux de déroulement des mobilisations, mais aussi probablement aux dispositions sociales et culturelles des agriculteurs de cette période, qui produisent des effets sur les formes de mobilisation. Les paysans sont incités sensiblement à la modération de leurs méthodes de protestation par leur environnement. Lorsqu'ils élaborent leur stratégie d'action, ils doivent dorénavant tenter de combler la perte d'une ressource essentielle. Ils sont en effet confrontés, en 1983 et 1984, à une contrainte structurelle liée à la dépopulation du monde agricole ${ }^{30}$. En conséquence, ils peuvent difficilement dans le cadre de leurs mobilisations se prévaloir de la légitimité du nombre. Il leur manque ainsi un des éléments essentiels qui, combiné avec "la volonté d'agir et la netteté du programme mobilisateur", permettent, selon Tilly, d'obtenir plus souvent satisfaction ${ }^{31}$. Se pose donc de manière de plus en plus sensible, la population agricole décroissant toujours régulièrement, le problème des soutiens extérieurs aux revendications paysannes, qui constitueront une ressource pertinente pour la réussite de leur contestation. Jusqu'au milieu des années 70 , les paysans ont fait prévaloir une logique de solidarité avec les ouvriers. Toute autre est celle qui influence les formes de mobilisations choisies dans les années 80 : les agriculteurs tentent désormais de s'attirer les faveurs de l'"opinion publique". Il est vrai que, depuis 1961, cette notion s'est imposée comme nouvelle variable qui transforme les situations d'interdépendance stratégique avec lesquelles les paysans doivent composer. Le jugement que "porte" l'opinion sur les revendications paysannes influe plus sensiblement sur le choix de leurs registres d'action. Son soutien constitue en effet, pour cette catégorie sociale dépourvue de capital économique et culturel, une ressource non négligeable de négociation, ainsi que l'a montré Lipsky ${ }^{32}$. Sans son 
"assentiment", leur contestation a de moindres chances de connaitre une issue favorable. Zimmermann a montré par ailleurs que là où les actions de contestation sont illégales, elles se doivent d'apparaître comme légitimes pour être acceptées par l'opinion publique $^{33}$. C'est pourquoi, il est essentiel de la convaincre de la légitimité de la cause défendue. D'où la nécessité de mettre en oeuvre des opérations de sensibilisation à l'adresse des journalistes, des élus, des citadins, etc., pour mettre fin à l'a priori défavorable que les violences ont contribué à donner des protestations. De fait, la recherche du soutien de l'opinion passe par la construction publique d'une image positive de soi. Ce travail de présentation de soi conduit les paysans à recourir plus fréquemment à des formes édulcorées de mobilisation. D'autres facteurs induisent l'auto-contrôle et l'évitement des modes de protestation jugés trop violents. Ce sont la multiplication des condamnations des violences paysannes, l'élargissement de la provenance de ces contestations, ainsi que l'abaissement de leur seuil d'apparition (à des violences moindres, s'oppose désormais une réprobation plus large). Alors que, presque seules, les autorités publiques s'élevaient auparavant contre ces modalités d'action, on les voit réprouvées en 1983 et 1984 par des syndicats (CGT, CFDT, FO, SNUI, FEN, et même SNPT) et des partis politiques ${ }^{34}$ mais aussi, de manière plus surprenante, par le ministre britannique de l'agriculture, les agriculteurs et les routiers belges, le Parlement européen et le gouvernement néerlandais ${ }^{35}$, etc. Ces quelques exemples de contempteurs des violences paysannes signalent combien l'agriculture française est désormais intégrée à l'économie mondiale; les agriculteurs sont insérés dans des relations d'interdépendance qui les contraignent à une plus grande maîtrise de leurs actions $^{36}$. De fait, les paysans ne construisent pas leurs stratégies d'action dans un vide social. Les réactions, positives ou négatives, de l'environnement dans lequel s'inscrit leur mobilisation, influent sur le modelage et le remodelage de leur registre d'action. Les oppositions croissantes à leur violence contribuent à rendre contre-performante la mise en oeuvre de certaines actions directes. Aussi les paysans furent-ils incités ces années là à adopter des comportements plus modérés: c'est ce qui explique la croissance des consignes de calme et de modération provenant des syndicats et l'élaboration d'actions voulues comme non violentes, afin d'estomper les images contraires données auparavant. Dans cette perspective, les paysans ont organisé des campagnes d'"explication" ou de "sensibilisation de l'opinion publique" auxquelles ils ont donné une visibilité maximale. Egalement, ils ont programmé, dans un souci "d'impact maximum sur les media", des manifestations d'agricultrices ${ }^{37}$, tablant sur une "division (sexuelle) du travail entre manifestations violentes exclusivement masculines et manifestations symboliques (de femmes)" ${ }^{38}$. Mais les mobilisations se caractérisent également, à l'initiative des paysans eux mêmes, par une certaine modération et une tendance à la symbolisation des actions. Les protestations de 1983 et 1984 se différencient en effet de celles de 1961, du point de vue de leur configuration générale. L'établissement du rapport entre le nombre de journées d'actions et la durée de ces deux mouvements, montre que leur intensité est moindre : les paysans mettent en oeuvre des actions collectives seulement un jour sur deux en 1983, et un jour sur trois en 1984 (contre 3 jours sur 4 en 1961). D'autre part, on n'observe pas, comme en 1961, un semblable crescendo dans le déroulement du conflit. Bien au contraire, en 1983 et 1984 se succèdent périodes d'actions et périodes de latence, qui peuvent parfois s'étaler sur une semaine entière. On voit donc qu'en aucune manière ces deux mouvements ne se sont inscrits dans une logique de conflit frontal visant, par un déploiement incessant d'activités, la "reddition" de l'ennemi. On peut même supposer 
que ces mouvements s'inscrivent d'emblée dans une logique globale de marchandage, les hommes politiques ayant généralement quelques réticences à ouvrir des discussions "sous la pression de la rue". Cette remarque pourrait sembler invalidée par les nombreuses négociations qui ont eu cours en $1961^{39}$. Il est vrai que tous les conflits, même les plus violents, donnent lieu à des discussions entre responsables syndicaux et représentants des pouvoirs publics. Mais ce qui varie entre les deux types de protestation, intense et diffuse, c'est la possibilité qu'offrent les secondes de mise en scène publique des négociations ${ }^{40}$, qui pourront contribuer à donner une image plus déconflictualisée de la contestation. Cette représentation pourra produire en retour des effets modérateurs sur la mobilisation. Les paysans, soucieux de conserver auprès des autorités publiques un capital de légitimité, s'efforceront alors davantage d'éviter toute démonstration susceptible de faire achopper les négociations, tout débordement violent risquant de saper l'image de sérieux acquise par les interlocuteurs officiels des pouvoirs publics. Les dirigeants paysans ont, en effet, un intérêt évident à faire montre de leur capacité à contrôler le comportement de leur base. D'autre part, l'élaboration concertée de mesures en faveur de l'agriculture à laquelle donnent lieu les discussions entre syndicats et gouvernement, est à même de fournir aux paysans des gratifications symboliques, qui confortent leur sentiment "d'être entendus"41, et dissuadent les paysans de recourir aux méthodes violentes. Les protestations paysannes sont par ailleurs plus souvent symboliques. On pense ici aux actions de "communication" qui sont basées sur l'explicitation des revendications. Cette démarche témoigne d'une modération très sensible des formes de protestation, en comparaison des actions violentes bien évidemment mais également de celles qui reposent sur l'usage de la force. En 1983, les paysans ont ainsi recouru à une forme originale d'expression de leurs motifs de mécontentement : ils avaient mis au point un film expliquant les effets négatifs des montants compensatoires monétaires qu'ils offrirent aux députés à l'occasion d'une manifestation devant l'assemblée nationale, ou aux représentants des pouvoirs publics, après avoir fait irruption dans des locaux officiels. Cette intention de communiquer est désormais, de manière plus explicite, au fondement de nombreuses actions, comme les pénétrations dans des lieux publics. Egalement, les paysans tentent d'administrer la preuve de la légitimité de leurs préoccupations : lorsqu'ils découvrent des produits importés, ils les apportent comme pièces à conviction à des élus ou représentants des pouvoirs publics afin de leur démontrer que les MCM rendent plus aisées des importations qui leur créent une concurrence déloyale: "Les gens de l'Etat n'entendaient pas nos principes mathématiques, comme quoi on allait à la faillite si les MCM restaient (..) C'était à nous d'apporter la preuve de ce que l'on voulait expliquer"42. A cela s'ajoutent des actions opérant une véritable mise en scène de la protestation. On pense cette fois aux dons d'un franc symbolique au ministre des finances qui ont été faits après l'investissement de centres des impôts ${ }^{43}$, mais aussi à la "caravane avicole et anti-MCM" qui défila dans toute la Bretagne avant de se rendre à Paris puis à Bruxelles. C'est également le cas du défilé organisé autour d'un camion d'importation, utilisé comme "pièce à conviction" : partant de Bretagne, il devait être escorté par des paysans toujours plus nombreux au fil de son trajet, pour arriver enfin à Paris, orné de banderoles, dans un concert de klaxons, et être exposé place des Invalides. Ces formes d'actions sont selon nous significatives donc d'une évolution tendancielle de la contestation paysanne vers la modération. 


\section{NOTES}

1. Ouest-France, 3 octobre 1967.

2. Ouest-France, 18 juillet 1974.

3. Le Monde, 24-25 avril 1983.

4. Cette définition est empruntée à Graham (H.D.) et Gurr (T.), The History of Violence in America, New-York, Bantham Books, 1969, p. 32.

5. Pour l'analyse de cette manifestation, voir Champagne (P.) "La manifestation, la production de l'événement politique", Actes de la Recherche en Science Sociale, $\mathrm{n}^{\circ}$ 52-53, Juin 1984, pp.19-41.

6. A partir d'une analyse exhaustive des manifestations paysannes sur dix ans, Marc Pinol remarquait que c'est en Bretagne et dans le Midi que se sont déroulées les protestations les plus violentes. Pinol (M.) "Dix ans de manifestations paysannes sous la cinquième République (1962-1971)", in Revue de Géographie de Lyon 50(2), 1975 p. 119 et suiv. Concernant plus particulièrement le Midi Viticole, voir les analyses de Patrice Mann, L'activité tactique des manifestants et des forces mobiles lors des crises viticoles du Midi (1950-1990). Contribution à l'étude de la dynamique des répertoires d'actions collectives, Rapport réalisé pour le compte de l'IHESI, Septembre 1991 ; voir également Alain Guillemin, "Montredon : intolérables violences ou violence de l'intolérable" in Les agriculteurs entre la politique et la politique agricole, Actes du colloque AFSP Les agriculteurs et la politique depuis 1970,1987, reprographié. Du même auteur, l'analyse de la séquestration d'Edith Cresson "Doucement, c'est tout de même une femme. Remarques sur le statut de la violence dans les manifestations paysannes", in Actes de la Recherche en Science Sociale, ${ }^{\circ} 52-53$, juin 1984, pp. 42-48.

7. Elias (N.), La civilisation des moeurs, (1939), Calmann Lévy, Agora, 1990.

8. Sur le concept de répertoire d'action, voir Charles Tilly, La France conteste. De 1600 à nos jours, Fayard, 1986, p. 541 et suivantes.

9. On entendra par mouvement, toute contestation donnant lieu à de multiples actions collectives en vue d'obtenir satisfaction sur des revendications agricoles susceptibles de faire l'objet d'une solution politique. On différencie les mouvements paysans des manifestations ponctuelles qui ne se déroulent pas sur une longue période. Cette définition rejoint celle que donne Tilly du mouvement social comme "série de défis aux autorités, surtout nationales" in La France conteste..., opus cit., p. 543.

10. Pour une discussion sur les difficultés d'ordre méthodologique inhérentes à notre corpus et à l'objectivation de la violence, se reporter au texte de notre rapport au IV ${ }^{\circ}$ Congrès de l'AFSP, Table ronde $\mathrm{n}^{\circ} 1$ "La violence politique dans les démocraties européennes occidentales", 23-26 septembre 1992.

11. Favre (P.), La manifestation, FNSP, 1990, p. 15.

12. Un exemple extrême de ce type de précautions nous est donné par la manifestation organisée à Paris par la FNSEA en 1982. Voir à ce sujet P. Champagne, op. cit., p. 23 et suiv.

13. Notre distinction rejoint celle que Graham et Gurr ont établie entre violence et force, au sens où cette dernière "implique la menace sinon l'usage actuel de la violence", op. cit., p. 32.

14. Philippe Braud, Le jardin des délices démocratiques, FNSP, 1991, p. 140.

15. Philippe Braud, ibid, p. 140. 
16. Charles Tilly, "Les origines du répertoire de l'action collective contemporaine en France et en Grande-Bretagne", XX ${ }^{\circ}$ siècle, 1984, p. 94.

17. Si tant est que cela ait une signification. On pouvait établir à 116 le nombre de lignes téléphoniques détériorées dans les années 60 , et à 5 le nombre de déprédations à l'encontre de bâtiments et de véhicules, soit un total de 121. Les déprédations lors de barrages et d'investissements depuis 1972 s'élèvent à 62, et les destructions ont touché 18 camions en 1983 et 1984 (elles sont pour 1972 pas chiffrables). Mais rapprocher les détériorations téléphoniques des déprédations pose le problème du sens de cette comparaison. On ne prétend absolument pas établir une quelconque analogie, mais seulement signifier que la croissance des déprédations, lors de barrages et d'investissements, invalide la première hypothèse posée, concernant la modération des protestations.

18. Mann (P.), "Répertoire des modes d'action paysans : continuité et ruptures", Les agriculteurs entre la politique et la politique agricole, Actes du colloque AFSP Les agriculteurs et la politique depuis 1970, 1987, reprographié, p. 2 et suivantes.

19. Charles Tilly, La France conteste, op. cit., p. 543.

20. Sur les enjeux syndicaux de ce mouvement, voir Yves Tavernier, "Le syndicalisme paysan et la politique agricole du gouvernement. Juin 1958-avril 1962", in RFSP, vol. XII, $n^{\circ} 3$, Septembre 1962, p. 599 et suiv. Les enjeux de ce mouvement et son déroulement sont analysés également par Henri Mendras et Yves Tavernier, "Les manifestations de juin 1961", in RFSP, vol. XII, n 3, Septembre 1962, p. 647 et suivantes.

21. Pour une analyse économique de la situation agricole, qui motive cette revendication d'une organisation des marchés, voir Suzanne Quiers-Valette, "Les causes économiques du mécontentement des agriculteurs français en 1961", in RFSP, vol. XII, n³, Septembre 1962, p. 555 et suivantes.

22. On trouvera des descriptions de l'investissement de la sous-préfecture de Morlaix, assorties de témoignages de leurs principaux protagonistes, dans les ouvrages de Fanch Elegoet, Révoltes paysannes en Bretagne, à l'origine de l'organisation des marchés, Ed. du Léon, 1984, p. 246 et ss ; Alain Baudoin et Louis-Roger Dautriat, Alexis Gourvennec, paysan directeur général, Fayard, 1977, p. 148 et suivantes.

23. Entretien avec Pierre Abéguilé, le 10 novembre 1992. En 1961, P. Abéguilé était secrétaire général de la section fermiers métayers de la FNSEA.

24. Cité par Fanch Elegoet, Révoltes paysannes en Bretagne, à l'origine de l'organisation des marchés, Ed. du Léon, 1984, p. 247.

25. Entretien avec Pierre Abéguilé, le 10 novembre 1992.

26. Sur la réorganisation syndicale et le quadrillage, cf F. Elegoet, op. cit., pp. 119-145 ;

A. Baudoin, L-R Dautriat, op. cit., pp. 48-58, ainsi qu'un article de François-Henri de Virieu in Le Monde, du 23 janvier 1960.

27. Cité par Fanch Elegoet, op. cit., p. 246.

28. Le Monde du 18-19 juin 1961.

29. Henri d'Halluin, dit Dorgères, constitua un Front Paysan, dans les années 30, et réussit à rassembler 400000 adhérents, en 1939, dans ses comités de défense paysanne. Son mouvement visait la restauration de la société, en instaurant le pouvoir de la paysannerie. Les méthodes d'action de ses comités étaient particulièrement et délibérément violentes.

30. L'INSEE recensait en 1962 plus de 3 millions d'agriculteurs exploitants, et seulement 1,3 million en 1985. Aussi les paysans sont-ils beaucoup moins en mesure de réaliser des mobilisations massives. Si les barrages regroupaient sans difficulté quelques milliers 
d'agriculteurs en 1961, les manifestations de 1967 rassemblaient environ 10000 paysans, l'une des plus importantes manifestations de 1983, le 6 mai, ne parvenait à mobiliser que 1500 personnes.

31. Charles Tilly, XXº siècle, 1984, op. cit., p. 95.

32. M. Lipsky, "La contestation comme ressource politique", in J. Padioleau, L'opinion publique, Mouton, La Haye, 1981, pp. 336-363.

33. Ekkart Zimmermann, Political Violence, Crises and Revolutions, Cambridge (Mass), Schenkman, 1983, p. 12.

34. A la suite de la destruction de la recette et du bureau d'enregistrement de l'hôtel des impôts de Carhaix, dans la nuit du 17 au 18 novembre 1983, l'intersyndicale des employés des impôts (CGT, FO, CFDT, SNUI) lançait un appel à manifester en signe de protestation (Le monde, 20-21.11.83). Le SNPT (Syndicat National des Policiers en Tenue) fit part de sa "profonde indignation devant cet acte de vandalisme", tandis que l'union départementale CGT dénonçait "une attitude haineuse et fascisante" (Ouestfrance, 21.11.83). Après le sac de la sous-préfecture de Brest, dans la nuit du 19 au 20 janvier 84, une manifestation de "défense de la démocratie" était également organisée, par les syndicats CGT, CFDT, FO, FEN, avec le concours du PS et du PC in Le monde, 26.01.84.

35. Les arraisonnements et séquestrations de véhicules d'importation de viande ont été à l'origine de ces critiques provenant de l'étranger : le ministre britannique de l'agriculture a "vivement protesté" à Bruxelles auprès de $\mathrm{M}$. Rocard et a menacé la France de mesures de rétorsion (Le monde 18.05.83) ; une centaine d'agriculteurs belges ont manifesté devant l'ambassade de France à Bruxelles (Ouest-France 12.01.84), tandis que des routiers belges bloquaient un poste frontière, suite au refoulement de 2 camions par les paysans français (Ouest-France 13.01.84). Le Parlement européen a quant à lui condamné leurs "actes de violence" (Le Monde 21.01.84). Le gouvernement néerlandais devait protester à son tour auprès des Communautés de Bruxelles (Le Monde 22-23.01. 84).

36. Sur les effets de l'interdépendance sur l'auto-contrôle et la maîtrise des pulsions, voir Norbert Elias La dynamique de l'Occident, (1939), Calmann-Levy, Agora, 1990, p. 181 et suivantes.

37. Ces opérations sont alors organisées à l'échelle nationale. Les 16 et 17 mai 1983, le CNJA et la FNSEA demandaient à leurs sections départementales de mettre en oeuvre des campagnes d'"explication des revendications à l'opinion publique". Le 16 mai également, des manifestations de femmes étaient organisées à Rennes, Vannes et Saint Brieuc. Celles-ci suivaient une manifestation à Saint Brieuc, le 6 mai, au cours de laquelle les paysans avaient systématiquement détérioré les aménagements de la ville. Le 3 décembre 1983 également, la FNSEA lançait un mot d'ordre de mobilisation, assorti de consignes expresses de "calme et de non violence". Les paysans mirent alors en place des barrages filtrants et distribuèrent à cette occasion des tracts ou des produits agricoles.

38. Martine Berlan, "Les paysannes dans la rue. Division du travail de manifestation dans l'agriculture depuis 1970", in Les agriculteurs entre la politique et la politique agricole, Actes du colloque AFSP Les agriculteurs et la politique depuis 1970, 1987, reprographié, p.9. Du même auteur, voir également "Farmers Wives in Protest ; a Theatre of Contradictions", in Sociologia Ruralis, 26(3-4), 1986. 
39. Le jour même de l'investissement de la sous-préfecture de Morlaix, par exemple, le sous-Préfet rencontrait les responsables de la FDSEA du Finistère, tandis que le lendemain, Michel Debré recevait les dirigeants de la FNSEA.

40. On trouvera une analyse des conséquences de "l'élargissement de la scène publique", depuis la mise en place des Conférences Annuelles Agricoles, sur les formes de mobilisation des paysans chez Patrice Mann, "Répertoire des modes d'action paysans : continuité et ruptures" in Les agriculteurs entre la politique et la politique agricole, Actes du colloque AFSP Les agriculteurs et la politique depuis 1970, 1987, reprographié, p. 14 et suivantes.

41. L'arrivée d'Edgard Pisani au ministère de l'agriculture en août 1961, et l'ouverture qu'elle a signifié à l'égard des revendications paysannes, en fournit une bonne illustration. Pendant plusieurs années après cette date, la Bretagne n'a connu que très peu de protestations violentes. Ce ministère $a$, de fait, favorisé la mise en oeuvre de nouvelles pratiques syndicales, qui privilégiaient la concertation.

42. Entretien avec J-J Riou, président du CDJA du Finistère en 1983, le 13. 11. 92.

43. A cette occasion, les paysans expliquaient que ce don visait à aider le ministère des finances à consentir l'effort financier que supposait le démantèlement des MCM qu'ils réclamaient.

\section{RÉSUMÉS}

De nombreux observateurs des protestations paysannes en France insistent sur leur caractère particulièrement violent. Les paysans sont alors présentés comme une exception en ce qu'ils échapperaient au processus général de modération des luttes qui affectent les conflits sociaux. cette thèse est réexaminée ici à partir de l'analyse comparative des mobilisations bretonnes qui se sont déroulées depuis 1960 jusqu'à nos jours. Une simple typologie des violences, fondée sur leur intensité, ne suffit pas à rendre compte de manière très probante d'une modération de la contestation. Les paysans bretons, tout en modifiant sensiblement leurs protestations n'ont certainement pas proscrit l'usage de la violence. Mais une analyse plus large des processus de mobilisation, et des représentations afférentes, dans le permet de prendre toute la mesure d'une évolution dans le mouvement de juin 1961 où domine une logique très fortement conflictuelle d'instrumentalisation de la violence où celle-ci joue un rôle central. Au contraire en 1983-1984, il apparaît que les paysans sont sensiblement incités à réduire la part de violence qui demeure dans leurs mobilisations, et ceci sous la "pression", notamment, d'une opinion publique hostile aux manifestations de brutalités. Il est donc permis de conclure à une évolution tendancielle de la contestation paysanne vers la modération.

\section{INDEX}

Index géographique : France

Mots-clés : émeutes, Mouvements sociaux, ruralité 